\title{
Specific heat study of magnetic excitations in a one-dimensional $S=1$ Heisenberg magnet with strong planar anisotropy
}

\author{
A. Feher, M. Orendáč, A. Orendáčová, and E. Čižmár \\ Department of Experimental Physics, Faculty of Science, P.J.Šafárik University, \\ Park Angelinum 9, 04154 Košice, Slovakia \\ E-mail: feher@kosice.upjs.sk
}

Received February 14, 2002

\begin{abstract}
The results of experimental studies of the specific heat of the magnetic chain compounds $\mathrm{Ni}\left(\mathrm{C}_{2} \mathrm{H}_{8} \mathrm{~N}_{2}\right)_{2} \mathrm{Ni}(\mathrm{CN})_{4}, \quad \mathrm{Ni}\left(\mathrm{C}_{11} \mathrm{H}_{10} \mathrm{~N}_{2} \mathrm{O}\right)_{2} \mathrm{Ni}(\mathrm{CN})_{4}$, and $\mathrm{Ni}\left(\mathrm{C}_{10} \mathrm{H}_{8} \mathrm{~N}_{2}\right)_{2} \mathrm{Ni}(\mathrm{CN})_{4} \cdot \mathrm{H}_{2} \mathrm{O}$ are reported. All compounds are identified as $S=1$ planar Heisenberg magnetic chains with large planar anisotropy and different values of the in-plane anisotropy constant. The low-temperature specific heat data are interpreted assuming the existence of noninteracting excitons and antiexcitons as elementary excitations from the singlet-ground state. The extended strong-coupling model is used for analysis of the data at higher temperatures. The applicability of the models used with respect to the value of the in-plane anisotropy is discussed.
\end{abstract}

PACS: 75.40.Cx, 71.20.Be, 75.50.Ee

\section{Introduction}

The physics of one-dimensional (1d) Heisenberg magnetic systems has been of interest during the last three decades, but particularly significant progress in both experiment and theory has been made during last several years. The failure of the description of the thermodynamic properties of $1 \mathrm{~d}$ magnetic systems in the framework of the spin-wave model demonstrated the importance of spin fluctuations and nonlinear effects in these systems [1]. For example, solitary excitations were used for the explanation of spin fluctuations in the systems possessing a Haldane gap [2], whereas out-of-easy-plane fluctuations from the singlet ground state, i.e. (anti)excitons, have been predicted theoretically in systems with strong planar anisotropy [3,4].

In this paper we report low-temperature specific heat results of the three magnetic chain compounds $\mathrm{Ni}\left(\mathrm{C}_{2} \mathrm{H}_{8} \mathrm{~N}_{2}\right)_{2} \mathrm{Ni}(\mathrm{CN})_{4}, \quad \mathrm{Ni}\left(\mathrm{C}_{11} \mathrm{H}_{10} \mathrm{~N}_{2} \mathrm{O}\right)_{2} \mathrm{Ni}(\mathrm{CN})_{4}$, and $\mathrm{Ni}\left(\mathrm{C}_{10} \mathrm{H}_{8} \mathrm{~N}_{2}\right)_{2} \mathrm{Ni}(\mathrm{CN})_{4} \cdot \mathrm{H}_{2} \mathrm{O}$ (hereafter abbreviated as NENC, NDPK, and NBYC, respectively). Our specific heat data measurements together with complementary data of susceptibility, magnetization, and ESR measurements, which were published elsewhere [5-7], have confirmed that NENC,
NDPK, and NBYC can be considered as a quantum $S=1$ magnetic chain with planar anisotropy and nonzero in-plane anisotropy. Specific-heat data were analyzed within the framework of the extended strong-coupling theory with in-plane anisotropy incorporated [8]. We focus our attention on the validity of this model with changing value of the in-plane anisotropy constant. Moreover, the specific heat of NENC and partially NDPK below $600 \mathrm{mK}$ can be interpreted assuming the presence of noninteracting (anti)excitons predicted in [3,4].

\section{Strong-coupling theory for $S=1$ planar magnetic chain}

The behavior of 1d $S=1$ Heisenberg-Ising model characterized by the Hamiltonian

$$
\begin{aligned}
H=\sum_{n=1}^{N}[ & -2 J\left(S_{n}^{x} S_{n+1}^{x}+S_{n}^{y} S_{n+1}^{y}+\right. \\
& \left.\left.+\lambda S_{n}^{z} S_{n+1}^{z}\right)+D\left(S_{n}^{z}\right)^{2}\right],
\end{aligned}
$$

where $n$ is the site index; $S_{n}$ are spin operators at the $n$th site; $J$ is the intrachain exchange interaction: $\lambda$ is the exchange interaction anisotropy constant, and $D$ characterizes the single-ion aniso- 
tropy. This Hamiltonian was studied numerically in dependence on the magnitude and sign of the parameters $\lambda$ and $D$ [9]. The $D$ vs. $\lambda$ phase diagram for the ground state of the Hamiltonian (1) has been constructed [10]. It is evident from Fig. 1 that the relatively simple Hamiltonian (1) possesses a variety of physically different ground state phases with quantum phase transitions between them.

The spectrum of the Hamiltonian (1) depends strongly on the value of the dimensionless parameter $\alpha=D /|J|$. Here we are interested in a so-called large- $D$ phase, for which $D / J>>1$ (grey area in the phase diagram on Fig. 1). For the large- $D$ phase the exchange Hamiltonian in (1) may be treated as a perturbation [3,4]. Consequently, for the strong-coupling approximation the Hamiltonian (1) can be transformed to the form

$$
\begin{gathered}
\frac{H}{D}=H_{0}-\frac{V}{\alpha}, \quad H_{0}=\sum_{n=1}^{N}\left(S_{n}^{z}\right)^{2}, \\
V=\sum_{n=1}^{N}\left[\frac{1}{2}\left(S_{n}^{+} S_{n+1}^{-}+S_{n}^{+} S_{n+1}^{-}\right)+\lambda S_{n}^{z} S_{n+1}^{z}\right] .
\end{gathered}
$$

In the limit $\alpha \rightarrow \infty(J=0)$, the ground state of the Hamiltonian (2), $H=H_{0}$, is given by the product of states with vanishing azimuthal spin $|0\rangle=|0,0, \ldots, 0\rangle$ ( $S_{n}^{z}=0$ for $n$ sites $)$. The ground state is nonmagnetic and disordered even at zero temperature. The first excited states with one nonzero azimuthal $\operatorname{spin}\left(S_{n}^{z}= \pm 1\right)$ for any $n$ are denoted as

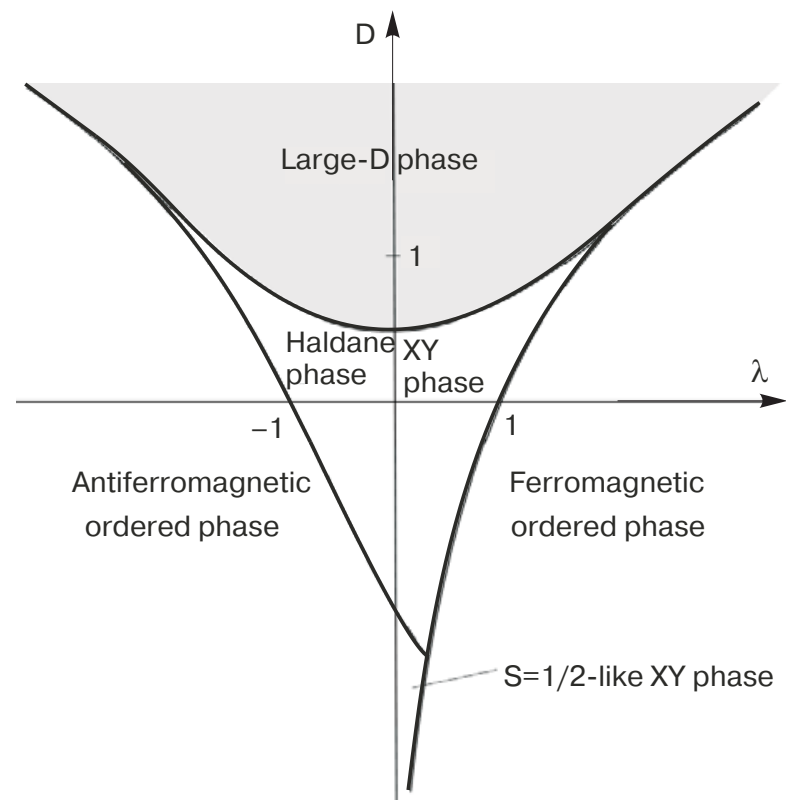

Fig. 1. $(\lambda, D)$ phase diagram for the ground state of Hamiltonian (1) for $S=1 . D$ and $\lambda$ are in the units of $J$ [10]. $|n\rangle$ and $|\bar{n}\rangle$, these are so-called exciton (e) and antiexciton $(\bar{e})$, respectively. Both $|n\rangle$ and $|\bar{n}\rangle$ are eigenstates of the unperturbed Hamiltonian $H_{0}$ for any $n$.

Higher excited states can be obtained by assigning the value $S_{n}^{z}= \pm 1$ to two or more sites with corresponding energies $2 D, 3 D, \ldots$ Generally, excited states fall into the bands of energies $i D$ corresponding to $i$ sites with nonvanishing azimuthal spins of both signs. These bands are highly degenerate in the limit $\alpha \rightarrow \infty$.

A finite and sufficiently small $J$ value may be considered as a perturbation which will transform the energy levels to bands of a finite width. The low-lying bands do not overlap for a sufficiently large $D / J$ ratio. The elementary excitations from the singlet-ground state carrying total spin momentum \pm 1 have been named excitons and antiexcitons, respectively. Perturbation theory to the third order [3] was used to derive a dispersion relation for a doubly degenerate (anti)excitonic mode, yielding:

$$
\omega_{k}=D\left[1+\omega_{1} / \alpha+\omega_{2} / \alpha^{2}+\omega_{3} / \alpha^{3}+k\right]
$$

where $\omega_{1}=-2 \cos (k), \omega_{2}=1+2 \sin ^{2}(k), \omega_{3}=$ $=1 / 2\left[1+8 \sin ^{2}(k)\right] \cos (k)-2 \lambda \sin ^{2}(k) ; k$ denotes the wave vector. In addition, the energies of two-site excitations fall into a two-body continuum. Near the Brillouin zone boundary, some bound states emerge smoothly from the continuum.

This exciton dispersion was used for the calculation of the specific heat at very low temperatures, within a dilute-exciton approximation (DEA). Since (anti)excitons obey Bose statistics, their specific heat contribution will be of the form

$$
C(T)=\frac{R}{\pi} \frac{d}{d T} \int_{-\pi}^{\pi} \frac{\hbar \omega(k)}{\exp \left(\hbar \omega(k) / \mathrm{k}_{B} T\right)-1} d k
$$

The analysis of NENC specific heat using DEA [5] showed that the theoretical approach $[3,4]$ requires further extension in three ways. First, to provide calculations of the specific heat that are valid beyond the low-temperature region. Second, to furnish a corresponding calculation of the magnetic susceptibility. Third, to include the effect of small in-plane anisotropy. All three problems have been addressed by Papanicolaou and Spathis in [8]. They carried out a systematic strong-coupling expansion to obtain analytical approximations for the spectrum of elementary excitations, the specific heat, and the magnetic susceptibility. A comparison of these results for a uniaxial $(\varepsilon=E / D=0$, where $E$ characterises the in-plane anisotropy) ferromagnetic chain with an intermediate easy-plane aniso- 
tropy $(\alpha=5)$ and numerical data calculated by Blöte [9] led to the following conclusions: the expanded strong-coupling approximation (ESCA) takes over decisively for temperatures larger than $T \sim 0.15 D / k_{B}$, where the DEA, in which effects from mutual interaction of excitons are neglected, begins to deteriorate. The value of the anisotropy restricts the applicability of the ESCA. For relatively low values of the easy-plane anisotropy in the region $|\alpha|_{\mathrm{cr}}<2.5$, the predictions of the ESCA begin to deteriorate gradually, and the disagreement between the theory and Blöte's numerical data increases. This deterioration becomes apparent by the formation of a kinklike anomaly on the low-temperature side of the specific heat. Therefore the results may be applied for $|\alpha|_{\mathrm{cr}}>2.5$ (for $\varepsilon=0$ ) but with caution at the lower end of this inequality. The in-plane anisotropy $(0<\varepsilon<1)$ causes a depression of the specific heat around the maximum and its enhancement at low temperatures. The latter is partly explained by the softening of one of the exciton modes at nonvanishing $\varepsilon$. For reasonably large values of $|\alpha|$ the theory requires a stronger condition for the value of the in-plane anisotropy $(\varepsilon<<1)$.

\section{Experimental details}

\subsection{Crystal structure}

NENC crystallizes in the monoclinic space group $P 2_{1} / n, a=7.104(3) \AA, b=10.671(3) \AA, c=$ $=9.940(2) \AA, \beta=114.68(2)^{\circ}$, and $Z=2$ [11]. The structure is built of neutral chains running along the $c$ axis. The repeating unit is $-\left[\mathrm{Ni}(\mathrm{en})_{2}-\mathrm{NC}-\mathrm{Ni}\left(\mathrm{CN}_{2}\right)-\mathrm{CN}\right]-$, where en $=\mathrm{C}_{2} \mathrm{H}_{8} \mathrm{~N}_{2}$, and two distinct nickel(II) sites are present. In the $\left[\mathrm{Ni}(\mathrm{CN})_{4}\right]^{2-}$ anion, the nickel is in a square planar configuration, being bonded to four cyano groups through $\mathrm{C}$ atoms. This nickel(II) ion is diamagnetic. In the $\left[\mathrm{Ni}(\mathrm{en})_{2}\right]^{2+}$ cation, the nickel is in a distorted octahedral configuration, where four $\mathrm{N}$ atoms of two en molecules are in the basal plane $\left(d_{\mathrm{Ni}-\mathrm{N}_{\mathrm{en}}}=2.107 \AA\right)$, while two $\mathrm{N}$ atoms from the cyano group are in apical positions $\left(d_{\mathrm{Ni}-\mathrm{N}_{\mathrm{en}}}=\right.$ $=2.089 \AA$ ). This nickel(II) ion is paramagnetic. For these paramagnetic ions, the intrachain $\mathrm{Ni}-\mathrm{Ni}$ distance is $9.94 \AA$, whereas the shortest interchain $\mathrm{Ni}-\mathrm{Ni}$ distance is $7.104 \AA$ (along the $a$ axis). The chain is therefore made of paramagnetic $S=1$ octahedral nickel(II) ions linked by diamagnetic square planar $\mathrm{NC}-\mathrm{Ni}(\mathrm{CN})_{2}-\mathrm{CN}$ units. The chains are well insulated from each other with no chemical bonding between them. Consequently, NENC presents all the structural features required for a good quasi-1d system.

NBYC crystallizes in the orthorhombic space group Pbcn with cell parameters $a=14.067$ (1) $\AA$, $b=10.1759(7) \AA$, and $c=15.755(1) \AA$. The structure consists of infinite zigzag chains containing two kinds of nickel(II) ions. The nickel(II) ion in the $\left[\mathrm{Ni}(\mathrm{CN})_{4}\right]^{2-}$ anion is square planar coordinated and thus diamagnetic. The nickel(II) ion in the $\left[\mathrm{Ni}\left(\mathrm{C}_{10} \mathrm{H}_{8} \mathrm{~N}_{2}\right)_{2}\right]^{2+}$ cation is paramagnetic and is located at the center of the distorted octahedron. Since the distance between the magnetic nickel(II) ions is about $10 \AA$, the direct exchange interaction will be of minor importance. Although the separation between the chains is comparable to the distances between the paramagnetic ions within the chain, more complicated interchain superexchange paths are expected to allow the formation of wellisolated magnetic linear chains in this compound. A detailed structure has been published in [12].

The detailed crystal structure of NDPK has not been determinated yet, but from infrared spectrum of the compound we can deduce the main structural features. Similarly as for NENC and NBYC, two kinds of nickel(II) ions are considered in the structure. More specifically, the nickel(II) ion surrounded by four $\mathrm{CN}$ groups is diamagnetic and the nickel(II) ion placed at the center of a distorted nitrogen octahedron is paramagnetic.

\subsection{Specific heat}

The specific heat measurements of powdered samples were performed in two experimental devices. For $50 \mathrm{mK}<T<2.5 \mathrm{~K}$, the dual-slope method was applied, using an Oxford Instruments dilution refrigerator. $\mathrm{A} \mathrm{RuO}_{2}$ thermometer (Dale RC 550 with nominal room-temperature value of $1 \mathrm{k} \Omega$ ) was calibrated against a commercial Lake Shore thermometer (model GR 200A-30), which served as the main thermometer. A silver wire (50 $\mu \mathrm{m}$ in diameter and $7 \mathrm{~cm}$ long) was used as a thermal link between the cold thermal reservoir and the platform containing the sample, the $\mathrm{RuO}_{2}$ thermometer, and a manganin heater. The resistance of the thermometer was measured by an ac $(72 \mathrm{~Hz})$ resistance bridge. The experimental data were corrected for the contribution of the thermometer, the manganin heater, and the varnish (GE 7031) used to anchor the powdered coin-shaped sample to the measuring platform. This additional contribution was measured in a separate run and was found to be maximum $10 \%$ of the total heat capacity. 
At higher temperatures, $2 \mathrm{~K}<T<10 \mathrm{~K}$, standard adiabatic calorimetry was used in a ${ }^{4} \mathrm{He}$ cryostat equipped with a mechanical heat switch. A $220 \Omega$ and $1 \mathrm{k} \Omega$ nominal value Allen Bradley thermometer was calibrated against the Lake Shore thermometer (GR-200A-1000CD) and served as the main thermometer in this arrangement. The weights of the samples used for the heat capacity measurements were: NENC - $800 \mathrm{mg}$, NBYC - $345 \mathrm{mg}$ and $1050 \mathrm{mg}$, and NDPK $-97 \mathrm{mg}$. The overall accuracy of the dual-slope data is better than $5 \%$, while a 3\% accuracy was achieved with the quasi-adiabatic technique.

\section{Results and discussion}

\subsection{NENC}

Heat-capacity measurements were performed in the temperature region from $50 \mathrm{mK}$ to $10 \mathrm{~K}$. Since NENC is a magnetic insulator, only lattice and magnetic contributions to the total specific heat are assumed for the present discussion. The lattice contribution was subtracted by finding the temperature region where the data may be satisfactorily described by the equation

$$
C_{T}(T)=\beta T^{3}+a T^{-2},
$$

where $\beta T^{3}$ represents the low-temperature lattice contribution in the 3d Debye approximation, while the $\alpha T^{-2}$ contribution describes the high-temperature behavior of the magnetic heat capacity. For temperature region $5 \mathrm{~K}<T<9 \mathrm{~K}$, a least-squares fit yielded $\alpha=(69 \pm 3.5) \mathrm{J} \cdot \mathrm{K} / \mathrm{mol}$ and $\beta=$ $=(3.55 \pm 0.15) \cdot 10^{-3} \mathrm{~J} /\left(\mathrm{K}^{4} \cdot \mathrm{mol}\right)$. The magnetic specific heat $C_{M}$ is characterised by a round peak with a maximum value of $C_{\max }=$ $=(5.85 \pm 0.05) \mathrm{J} / \mathrm{K} \cdot \mathrm{mol}$ at $T_{\text {max }}=(2.4 \pm 0.1) \mathrm{K}$ (Fig. 2). The magnetic entropy was calculated numerically in the measured temperature region, and standard approximations were used for high and low temperatures to cover the whole temperature interval. The calculation yielded $8.68 \mathrm{~J} / \mathrm{K} \cdot \mathrm{mol}$, which is close to the theoretical value $R \ln (2 S+1)=9.13 \mathrm{~J} / \mathrm{K} \cdot \mathrm{mol}$ for an $S=1$ system. This broad maximum, together with the absence of a $\lambda$-type anomaly at least down to $50 \mathrm{mK}$, indicates the presence of short-range correlations in the studied system. In the first approximation, if in-plane anisotropy is neglected $(E=0)$, our system can be described by Hamiltonian (1). The specific heat of a system represented by this Hamiltonian for $\lambda=1$ was calculated numerically by Blöte [9]. The best agreement between the numerical predictions and

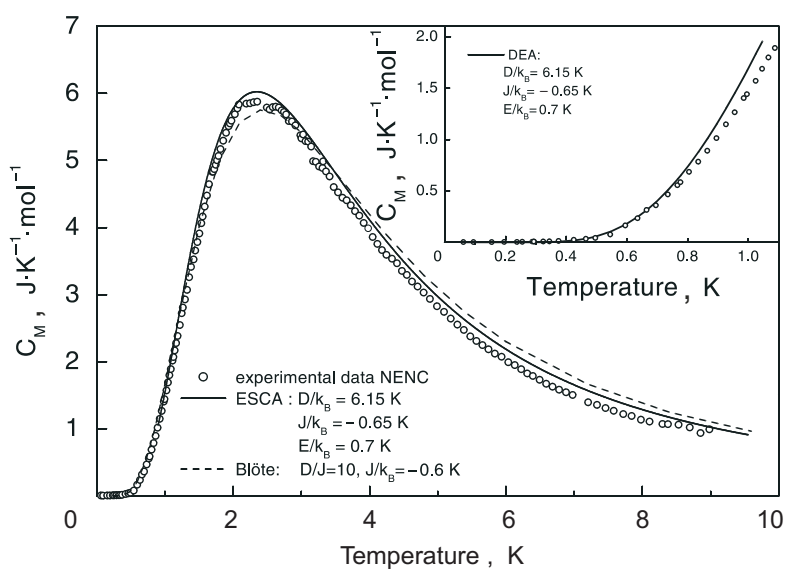

Fig. 2. The temperature dependence of the magnetic specific heat of NENC.

the experimental data was obtained for $D / k_{B}=6 \mathrm{~K}$ and $D /|J|=10-20$. The resultant $D /|J|$ ratio indicates that NENC can be considered a quantum $S=1$ system with strong planar anisotropy.

The sudden drop of $C_{M}$, observed below $1 \mathrm{~K}$, suggests the existence of a gap in the energy spectrum of the spin system, and this effect is most likely associated with magnetic anisotropy. The comparison of the low-temperature specific-heat data with the theoretical prediction for DEA (taking into account a small in-plane anisotropy), using $D / k_{B}=6.15 \mathrm{~K}, \mathrm{~J} / k_{B}=-0.65 \mathrm{~K}$ and $E / k_{B}=0.7 \mathrm{~K}$ is shown in the inset in Fig. 2. Clearly, the trend from $T<0.8 \mathrm{~K}$ is not followed at higher temperatures, since the DEA begins to fail due to exciton interactions. The temperature at which the excitonic interactions are significant in NENC $(T \sim 1 \mathrm{~K})$ coincides with the value $0.15 D / k_{B}=0.9 \mathrm{~K}$ estimated for a system with $D /|J|=5$ in the theoretical work [8]. The experimental specific-heat data above $1 \mathrm{~K}$ can be well described by the ESCA using the same set of parameters $D, J$, and $E$ (see Fig. 2). We can conclude that the magnetic specific-heat of NENC can be ascribed to the presence of low-lying elementary excitations, i.e., excitons predicted for large- $D$ systems in the framework of the strong-coupling theory.

\subsection{NDPK}

The experimental study of the specific heat of NDPK has been carried out with the aim of investigating a system located possibly close to the phase boundary between the Haldane phase and large- $D$ phase (Fig. 1) and to check the validity of the strong-coupling model for this system. Since the group $\mathrm{C}_{11} \mathrm{H}_{8} \mathrm{~N}_{2} \mathrm{O}$ is characterised by a different 
type of delocalization of charge density than $\mathrm{C}_{2} \mathrm{H}_{8} \mathrm{~N}_{2}$ in NENC, we have tried to change the surroundings of the paramagnetic nickel(II) ion with the expectation of obtaining a system with different anisotropy in comparison with NENC.

The specific heat of NDPK was measured in the temperature range from $100 \mathrm{mK}$ to $2.5 \mathrm{~K}$. A comparison of our results on the magnetic part of the specific heat with the numerical prediction of Blöte [9] gives $D / k_{B}=8.7 \mathrm{~K}$ and $D /|J|=5$ (Fig. 3). This result suggests that although the value of $D /|J|$ for NDPK is two times smaller than that for NENC, the system is still located in the large- $D$ phase rather than near the phase boundary. The specific heat data were analyzed using ESCA [8] with the aim of studying the influence of in-plane anisotropy $E$ as a potential source of the discrepancy between our results and Blöte's numerical predictions. Above temperature $1.2 \mathrm{~K}$ we found good quantitative agreement with the experimental data for $D / k_{B}=5 \mathrm{~K},|J| / k_{B}=0.94 \mathrm{~K}$ and $E / k_{B}=2.8 \mathrm{~K}$. Note that the value of $E(\varepsilon=0.56)$ indicates a large in-plane anisotropy in comparison with NENC. As was already mentioned in Sec. 2, the validity of the ESCA (for $E=0$ ) is limited to $|\alpha|_{\text {cr }}>2.5$ and $T>0.15 D / k_{B}$. The appearance of the kinklike anomaly of the artificial origin on the ascending side of the theoretical curve suggests a significant increase of $|\alpha|$ cr for large in-plane anisotropy.

Experimental data on the specific heat below $0.15 D / k_{B}$ were analysed in the framework of the DEA using the same set of values $D,|J|$, and $E$. It follows from the inset in Fig. 3 that the validity of the DEA is also restricted in the presence of large in-plane anisotropy in NDPK.

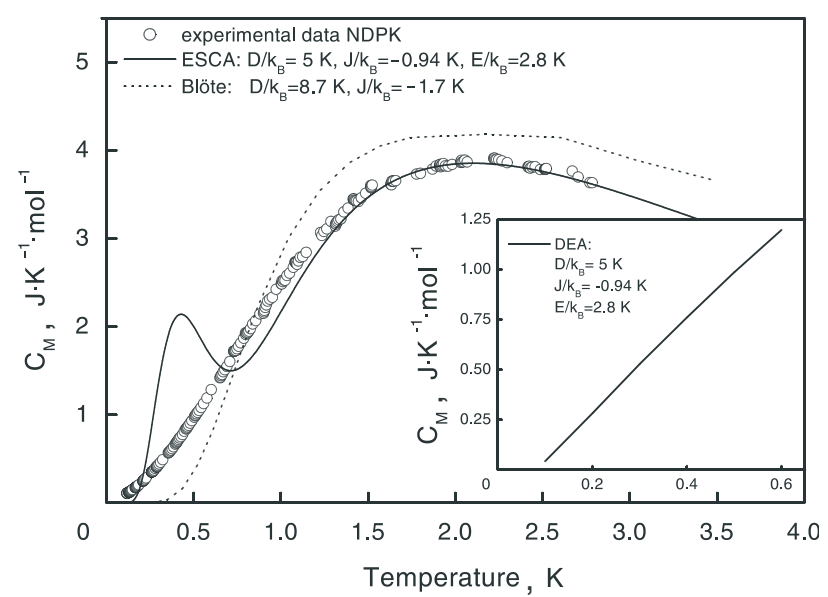

Fig. 3. The temperature dependence of the magnetic specific heat of NDPK.

\subsection{NBYC}

In addition we have tried to modify the magnetic anisotropy by replacing the group $\mathrm{C}_{11} \mathrm{H}_{8} \mathrm{~N}_{2} \mathrm{O}$ by $\mathrm{C}_{10} \mathrm{H}_{8} \mathrm{~N}_{2}$ with the aim of testing our conclusions on the validity of strong-coupling model on a system with strong in-plane anisotropy.

We have studied the heat capacity of NBYC in the temperature range from $100 \mathrm{mK}$ to $6 \mathrm{~K}$. The results of experimental studies of the magnetic part of the specific heat of NBYC are presented in Fig. 4. The broad maximum, together with the absence of a $\lambda$-type anomaly down to $100 \mathrm{mK}$, indicates a high degree of short-range order in this system. The magnetic specific heat was compared with numerical predictions of Blöte [9], where only $D$ and $J$ were involved.

The best agreement between the numerical predictions and the experimental data was obtained when $D / k_{B}=1.45 \mathrm{~K}$ and $D /|J|=1$. More detailed analysis of $C_{M}$ was carried out using the theoretical predictions with $D, J$, and $E$ taken into account in the ESCA [8]. In the temperature region $T>0.38 \mathrm{~K}$, the specific heat data can be satisfactorily described using the values $D / k_{B}=2.55 \mathrm{~K}$, $|J| / k_{B}=0.2 \mathrm{~K}$, and $E / k_{B}=1.5 \mathrm{~K}$. As in the case NDPK, the ESCA fails to describe the specific heat data below $T=0.15 D / k_{B}$ as can be seen in Fig. 4 . Moreover, we have tried to apply the DEA to describe the temperature behavior of the magnetic specific heat below the temperature $0.15 D / k_{B}$. As is evident from Fig. 4 (see the inset), the DEA fails to describe the specific heat data in the temperature range $100 \mathrm{mK}<T<380 \mathrm{mK}$. This result confirms that the validity of the DEA is restricted to low in-plane anisotropy.

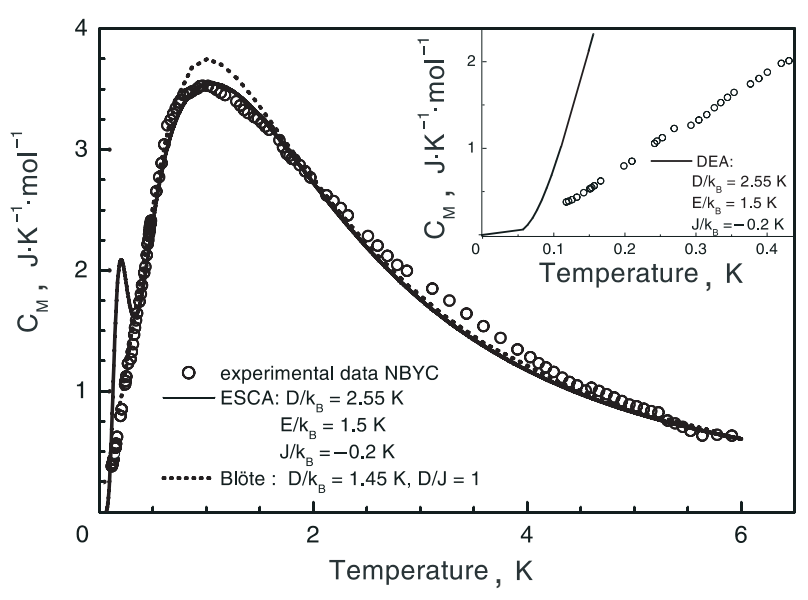

Fig. 4. The temperature dependence of the magnetic specific heat of NBYC. 
Values of the exchange constant and magnetic anisotropy constants

\begin{tabular}{|c|c|c|c|c|c|c|c|c|}
\hline \multirow[t]{2}{*}{ Compound } & \multirow[t]{2}{*}{$D / k_{B}, \mathrm{~K}$} & \multirow[t]{2}{*}{$|J| / k_{B}, \mathrm{~K}$} & \multirow[t]{2}{*}{$E / k_{B}, \mathrm{~K}$} & \multirow[t]{2}{*}{$\alpha=D /|J|$} & \multirow[t]{2}{*}{$E / J \mid$} & \multirow[t]{2}{*}{$\varepsilon=E / D$} & \multicolumn{2}{|c|}{$\begin{array}{c}\text { Model } \\
\text { (temperature range) }\end{array}$} \\
\hline & & & & & & & DEA $(\mathrm{mK})$ & ESCA $(\mathrm{K})$ \\
\hline NENC & 6.15 & 0.65 & 0.7 & 9.5 & 1.0 & 0.16 & 50-900 & $0.9-6$ \\
\hline NDPK & 5 & 0.96 & 2.8 & 5.2 & 2.9 & 0.56 & $100-600$ & $1.2-3$ \\
\hline NBYC & 2.55 & 0.2 & 1.5 & 12.7 & 7.5 & 0.59 & - & $0.38-6$ \\
\hline
\end{tabular}

\section{Conclusions}

Our specific heat studies have confirmed that NENC, NDPK, and NBYC can be considered as a quantum $S=1$ Heisenberg chain with strong planar anisotropy and nonzero in-plane anisotropy.

Magnetic specific heat data were analyzed within the framework of the strong-coupling theory [8] for both noninteracting excitons $\left(T<0.15 D / k_{B}\right)$ and expanded strong-coupling approximation $\left(T>0.15 D / k_{B}\right)$. The values of the exchange and magnetic anisotropy constants as well as their mutual ratios for the systems studied are summarized in the Table. We found that the region of the validity of ESCA for $|\alpha|_{\text {cr }}>2.5$ is in agreement with the predictions of Papanicolaou and Spathis, i.e., for $E=0$ it is valid for temperatures larger than $0.15 D / k_{B}$ and below this temperature a kinklike anomaly of artificial origin occurs on the ascending side of the specific heat curve for a system with strong in-plane anisotropy. The validity of the DEA is drastically suppressed with increasing value of in-plane anisotropy. We have observed very good agreement between predictions of DEA and specific-heat data only for NENC, which is characterized by a low $E / D$ ratio. In addition, on the basis of the results obtained we propose that in-plane anisotropy should be considered in any attempt to find a compound located close to the boundary between the Haldane and large- $D$ phases.

\section{Acknowledgments}

We are grateful to Prof. V. V. Eremenko for introducing our Košice group to the wonderful world of low-dimensional magnetism many years ago. One of us (A. F.) acknowledges the hospitality of CRTBT CNRS Grenoble during the preparation of this manuscript. Partial financial support for this work was provided by the grant No. $1 / 7473 / 20$ from the Slovak Ministry of Education. The material support of U. S. Steel DZ Energetika is gratefully acknowledged.

1. R. Steiner, J. Appl. Phys. 67, 5593 (1990).

2. F. D. M. Haldane, Phys. Lett. 93, 464 (1983).

3. N. Papanicolaou and P. Spathis, J. Phys. Condens. Matter 1, 5555 (1989).

4. N. Papanicolaou and P. Spathis, J. Phys. Condens, Matter 2, 6575 (1990).

5. M. Orendáč, A. Orendáčová, J. Černák, A. Feher, P. J. C. Signore, M. W. Meisel, S. Merah, and M. Verdaguer, Phys. Rev. B52, 3435 (1995).

6. M. Orendáč, T. Herrmannsdoerfer, A. Feher, and F. Pobell, Czech. J. Phys. 46, 1941 (1996).

7. M. Orendáč, E. Čižmár, A. Orendáčová, J. Černák, A. Feher, M.W. Meisel, K. A. Abboud, S. Zvyagin, M. Sieling, T. Rieth, and B. Lüthi, Phys. Rev. B61, 3223 (2000).

8. N. Papanicolaou and P. N. Spathis, Phys. Rev. B52, 16001 (1995).

9. H. W. J. Blöte, Physica B79, 427 (1975).

10. R. Bottet, R. Jullien, and M. Kolb, Phys. Rev. B28, 3974 (1983).

11. J. Černák, J. Chomič, D. Baloghová, and M. Duhaj-Jurčo, Acta Crystallogr. C44, 192 (1983).

12. J. Černák, and K. A. Abboud, Acta Crystallogr. C56, 783 (2000). 